\title{
A PROPÓSITO DE LA NOCIÓN DE MOVIMIENTO: VIRTUALIZACIÓN DE LOS MOVIMIENTOS SOCIALES
}

\author{
Juan Carlos Aceros, Sandra Coronado, Sayani Mozka y Vanessa Gamero
}

Universitat Autònoma de Barcelona

\section{Resumen}

Este trabajo intenta problematizar el concepto de "nuevos movimientos sociales". Hasta el presente, dicho esfuerzo se ha orientado especialmente a cuestionar el calificativo de "nuevo". Sin desconocer in dicho debate, nosotros tomamos otro camino: revisamos la palabra "movimiento" tratando de determinar aquello que se mueve en los movimientos sociales. Empleando a Nodo50 como caso y los postulados de Pierre Levy sobre la "virtualización" reflexionamos en torno a la posible transformación de los movimientos sociales contemporáneos, a partir del uso que éstos hacen de las tecnologías de la comunicación y la información (TICs). De esta manera, esperamos determinar si el concepto propuesto por Alberto Melucci deviene otro, se desterritorializa o problematiza en la llamada "Sociedad de la Información".

Palabras clave: Movimientos Sociales, Virtualización, Sociedad de la Información, TICs

\begin{abstract}
This paper intends to bring into question the concept of "new social movements". So far, the above-mentioned effort has oriented itself especially in putting in doubt the qualifying item of "new". Without ignoring this debate, we take another path: we revise the term "movement" and try to determinate what exactly it is that might move in social movements. By using Nodo50 as our main case and Pierre Levy's postulates on the "virtualisation" as reference mark, we reflect on the possible transformation of contemporary social movements starting from the use they make of Information and Communication Technologies (ICTs). Thus we hope to determine whether Alberto Melucci's concept turns into another one in the socalled "Information Society".
\end{abstract}

Keywords: Social Movements, Virtualisation, Information Society, ICTs.

\section{Introducción}

Las sociedades contemporáneas gestionan una alta densidad de información y lo hacen a velocidades inimaginables unas décadas atrás. En efecto, la información se ha convertido en recurso de la vida social (Melucci, 1999), sobrepasando los límites de la teoría matemática, de la comunicación, e interesando a la economía, la lingüística, la antropología, la sociología, la psicología social y también a los "movimientos sociales".

Al pensar en la "información" no nos referimos a la transmisión de mensajes desde un punto a otro, sino al contenido y los procesos de conocimiento y de acción social que surgen en el contexto de las interacciones entre personas y grupos. Informar se entiende, entonces, como un conjunto de prácticas orientadas a la generación e intercambio social de códigos, lenguajes, sentidos y, en 
términos amplios, cultura. La Sociedad de la Información viene siendo, por esta vía, un orden que se realiza cuando estas prácticas adquieren alcance planetario.

En la Sociedad de la Información, el poder es la capacidad de dar forma a la realidad organizando el flujo de información; es la capacidad de in-formar (Melucci, 1999, Zubero, 2004). Dicha capacidad, aseguran algunos autores, no es ejercida por todos de la misma forma: sólo unas pocas empresas y medios de comunicación pueden informar a nivel global. Este hecho, se afirma, viene acompañado por la distribución de una visión del mundo restringida y homogénea (Marí Sáez, 2004a); así como por la exclusión de miradas que no cuentan con los mismos medios para hacerse visibles (Zubero, 2004).

Los movimientos sociales reclaman, entonces, más espacios para in-formar y de esta manera incidir en la configuración del tejido social. Y lo hacen apostando por la incorporación de las tecnologías de la información y la comunicación (TICs) con una intensidad que varía de un colectivo o "movimiento" a otro. Algunos apuestan por la conectividad y por la dotación de los recursos necesarios; otros incluyen la alfabetización tecnológica. Existen unos más que emplean Internet para la denuncia y la protesta. Estos últimos pueden ver reforzada su forma de organización como ha ocurrido con los ecologistas, anarquistas y las feministas, sin embargo con mayor frecuencia han adoptado modelos reticulares que les eran ajenos (Marí Sáez, 2004a, 2004b). Finalmente, ciertos "movimientos" han buscado explicitar las lógicas que acompañan a la tecnología y reflexionan en torno a su relación con el cambio social y con sus agendas. Este nivel de apropiación de las TICs incluye también esfuerzos por adaptar las herramientas a los colectivos, así como para construir otras nuevas (Marí Sáez, 2004a).

Las implicaciones de este panorama son tan variadas como heterogéneas las propuestas de incorporación tecnológica. Aquí no las exploramos todas; nos centramos específicamente en una cuestión de carácter conceptual. Nos interesa saber cómo se transforma lo que entendemos por "movimiento social" cuando entran en escena las TICs. Por esta vía, no queremos aportar al debate sobre las diferencias entre el conflicto de clases y las actuales formas de acción colectiva. Lo que esperamos es identificar aquello que se mueve en estas últimas.

En resumen, nuestra comunicación es una reflexión sobre la noción de "movimiento". Para adelantarla, partimos del punto de vista de Alberto Melucci sobre la acción colectiva, así como de los postulados de Pierre Lévy sobre la "virtualización". Aplicamos estas miradas a un caso concreto: Nodo50, un Proveedor de Servicios de Internet para los "movimientos sociales". De esta manera intentamos contribuir al análisis de la acción colectiva en la Sociedad de la Información.

\section{De los movimientos a los sistemas de acción}

Examinar la idea de "movimiento social" desde Melucci, supone de entrada, ir más allá de los límites que la misma noción ha demarcado; plantearnos en todo caso desde una frontera conceptual, otras maneras de pensarla. Hoy, al menos dos elementos distinguen las formas de acción colectiva: multiplicidad y variabilidad. Reconocer estas características nos llevan a situar el fenómeno de los "movimientos sociales" fuera de las explicaciones mecanicistas y totalizantes distintivas de la modernidad. 
Nuestro análisis quiere superar la noción "nuevos movimientos sociales" y en esta apuesta admitimos que "algo" quedó sin explicación. En primer término, descolocamos el adjetivo no por un mero gusto lingüístico, sino porque nos parece necesario dilucidar la complejidad social distintiva de nuestro tiempo y discontinua de la modernidad; en ese sentido, los "movimientos sociales" actuales nos refieren a la vez, singularidad y complejidad, y la necesaria apuesta por un cambio en las formas de enunciarlos. En segundo lugar, conviene aclarar que tomamos distancia de aquellos estudios que insisten en el debate de lo novedoso, pues tal manera de definirlos, cumplían con el único objetivo de comparar las formas históricas del conflicto de clases (Melucci, 1999) respecto al nuevo momento en que la acción colectiva sucede. Así, tanto los análisis del movimiento obrero en una primera etapa, como después el de los movimientos más de tipo cultural: jóvenes, feministas, ecologistas en el que se enmarcó el "paradigma de lo novedoso", aluden a sujetos colectivos específicos. Melucci (1996, 1999) pone el acento en este aspecto, y señala el error epistemológico de los estudios que se han centrado en hacer de los "movimientos sociales," objetos empíricos o personajes históricos unitarios actuando sobre el sistema político.

No se trata pues, de desconocer a esos actores sociales históricos, ni su formas de acción. En todo caso, nos apartamos de la búsqueda de "nuevos movimientos sociales" y pensamos que un terreno más fértil, es el de preguntarnos por la forma como la acción plantea una novedad constante en la sociedad. Entendiendo que la "novedad" no es el atributo de un "objeto", sino un fenómeno que emerge en redes difusas, dinámicas y heterogéneas. En ese sentido, vemos la acción colectiva como un sistema abierto en el cual convergen una composición de elementos que le dan sentido y a la vez, crean lo social. En dichas formas de acción se involucran distintos niveles de la estructura social y sus componentes están relacionados con periodos históricos y actores sociales diversos. Melucci (1999) prefiere pensar así, en "sistemas de acción". Examinemos a continuación, algunas características asociadas a esta idea:

a. Los sistemas se acción cuentan con estructuras, es decir, con la integración e interdependencia de individuos y grupos. Así comprendidos, son "redes de solidaridad" construidas por objetivos, creencias, decisiones e intercambios, que operan en un campo sistémico.

b. Estas redes conforman una "identidad colectiva" a partir de la definición compartida, negociada del campo de oportunidades y constricciones ofrecidas a la acción colectiva. En otras palabras, los sistemas de acción son "construcciones sociales" que se estructuran en un contexto y gracias a intercambios y conflictos que ocurren en la interacción cotidiana. En dicha interacción se concretan orientaciones y propósitos plurales; ocurre una producción continua de relaciones sociales.

c. Los sistemas de acción detentan un poder que no es sólo político. Para Melucci (1999), las relaciones sociales son más amplias que las políticas, y los "movimientos" afectan primordialmente a las primeras. Por esta vía, impactan al sistema como un todo denunciando y alterando la lógica de la sociedad. "Anuncian los cambios posibles, no en el futuro distante sino en el presente de nuestras vidas; obligan a los poderes a mostrarse y les dan una forma y un rostro; utilizan un lenguaje que parece exclusivo de ellos, pero dicen algo que los trasciende y hablan por todos nosotros" (Melucci, 1999:11).

Estos tres referentes de los sistemas de acción son recíprocos con las tres dimensiones analíticas de la acción colectiva que nos sugiere Melucci $(1996,1999)$ : a) el establecimiento de lazos de solidaridad en tanto capacidad de los actores colectivos de reconocerse a sí mismos y de ser reconocidos como integrantes de un mismo sistema de relaciones, b) la presencia del conflicto que 
habla de un campo en disputa por dos adversarios, y c) la ruptura de los límites del sistema en el que se produce ese antagonismo.

Los "sistemas de acción" son, a un tiempo, medios y mensajes. En el primer sentido son redes que actúan como canales de información sobre los nuevos patrones de desigualdad y las nuevas formas de poder. En el segundo, son en sí mismos por su forma de actuar, una expresión de los conflictos sociales. Por tal razón, en la perspectiva de Melucci es menos importante lo que los "movimientos" dicen de sí mismos que el modo como los actores constituyen su acción (Melucci, 1999). Más aún, es significante lo que estos actores colectivos revelan sobre la realidad social.

Distinguimos en el concepto de Melucci $(1996,1999)$ dos constantes que nos sirven para pensar en los "movimientos sociales" como acontecimientos. En primer lugar, el campo dinámico de las interacciones cotidianas que estructuran los "sistemas de acción" y construyen su identidad. En segundo lugar, el conflicto que generan al interior del sistema social, a partir de la puesta en evidencia de sus tensiones y desigualdades, y la generación de un espacio en disputa. Asumimos que estos dos elementos se relacionan directamente con los polos latente y visible de la acción colectiva (Melucci, 1999), que intentamos resignificar enseguida empleando las ideas de Lévy (1999) en torno a la "virtualización".

\section{De los movimientos a los acontecimientos}

Hasta ahora hemos intentado cuestionar el concepto de "movimiento social". Empleando las ideas de Melucci $(1996,1999)$, nos acercamos a su desustanciación. En efecto, pasamos de un fenómeno empíricamente unitario a una red difusa y emergente. Ahora no tenemos un concepto que se refiere a un dato (lo dado), sino a un logro. En estas condiciones podemos emprender una reflexión sobre la acción colectiva como "acontecimiento".

Para adelantar dicha reflexión empleamos la obra de Pierre Lévy (1999). Este filósofo concibe que el acontecimiento es una forma de ser fecunda y potente, es el "ser como creación" (Pág. 123). Entendido como un éxodo ontológico creativo, el acontecimiento es una "heterogénesis" que lleva a "algo" a convertirse en otra cosa radicalmente diferente. Esta irrupción de la novedad se da en el devenir entre dos polos: uno latente, que Lévy denomina "virtual" y otro manifiesto, que recibe el nombre de "actual". Al respecto de su relación dialéctica nos dice el autor:

"Esencialmente problemático, lo virtual viene a ser una situación subjetiva, una configuración dinámica de tendencias, de fuerzas, de finalidades y de obligaciones que resuelven una actualización. La actualización es un acontecimiento en el sentido pleno del término. Un acto se ha cumplido aunque no esté predefinido en ninguna parte y aunque, en contrapartida, modifique la configuración dinámica en la que adquiere una significación." (Pág. 123)

Lo virtual, en este contexto, no es un producto informático o de la informática. No es una simulación de mundos ilusorios. Lévy (1999) recupera el concepto clásico, lo enriquece con aportes de académicos contemporáneos, y nos presenta lo virtual como una dimensión problemática de la realidad. Así, la virtualización debe ser entendida como la apertura de un campo conflictivo: nudo de tendencias o de fuerzas que inspiran los actos. La actualización por su parte, inventa una solución para el problema o una respuesta al conflicto planteado por lo virtual. Dicha (re)solución no está 
planteada en el enunciado problemático o en los términos del conflicto. De hecho, es la creación de una forma o idea nueva a partir de una configuración dinámica de fuerzas y finalidades.

En resumen, virtualidad y actualidad son dos maneras diferentes y complementarias de ser-comocreación (Lévy, 1999): lo virtual es un problema, lo actual su solución; la virtualización es apertura eterna de conflictos, la actualización es su proceso de resolución. El primer polo del acontecimiento es el porqué del segundo, y este último es el cómo del primero. Ambos pertenecen a la entidad considerada, son dos dimensiones del mundo humano1. En el caso de los llamados "movimientos sociales", lo virtual y lo actual equivalen a los polos latente y visible de los que habla Melucci (1999): mientras que uno crea nuevos códigos y permite que los individuos los experimenten, el segundo aparece cuando grupos entrelazados deciden enfrentar una cierta lógica social. De la misma forma que la pareja virtual-actual, estos polos no son mutuamente excluyentes, sino que se correlacionan: "La latencia alimenta la visibilidad con recursos de solidaridad y con una estructura cultural para la movilización. La visibilidad refuerza las redes inmersas. Proporciona energía para renovar la solidaridad, facilita la creación de nuevos grupos y el reclutamiento de nuevos militantes atraídos por la movilización pública que ya fluye en la red inmersa" (Melucci, 1999:74).

Conviene recordar que los polos latente/virtual y visible/actual tienen el carácter de acontecimiento. No son episodios que se sucedan cíclicamente. No se organizan uno después del otro hasta el infinito. No están instituidos. El ciclo, el proceso lineal y la institución son formas de fijar el acontecimiento y de expropiarle su novedad. Los límites que estos operadores plantean, son lo que supera constantemente la virtualización. Dicho logro se alcanza, al menos, a través de dos vías (Lévy, 1999): a. por "desterritorialización", es decir, desconexión de un medio o tiempo particular, y b. por el paso del interior al exterior y viceversa, por una inversión de los límites. Estos movimientos son eminentemente tácticos, pero ocurren de manera fractal, es decir, se presentan en diferentes niveles de la realidad. En el caso de los sistemas de acción social, lo virtual es la cotidianeidad de la latencia: está presente en la negociación y experimentación de los códigos culturales alternativos que se crean en este polo. La actualización de dichos códigos favorece su visibilidad y su distribución social: aquí los intercambios diarios se han organizado, se les ha otorgado un sentido y aparecen como realidades independientes y autosuficientes, es decir, como formas de vida alternativas de pleno derecho. Tal actualidad, que es más propia del polo visible, es a su vez virtualizante respecto al orden social con el cual se enfrenta: le pone en problemas, le cuestiona. Este hecho, como bien lo afirma Melucci (1999) es un signo de que la tensión y el conflicto son una constante en las sociedades complejas (al igual que ocurre en el nivel de las interacciones cotidianas del polo latente).

Además de fractal, la virtualización es relacional. No podemos decir que sea propia de los "movimientos sociales", sino que emerge en las interacciones que se dan en su polo latente, así como en sus vínculos con el contexto social o con el orden político. Esto es fundamental para entender el papel de la tecnología en la virtualización de los "movimientos sociales".

Los procesos de virtualización contemporáneos se ven acompañados por los desarrollos en materia de telecomunicaciones. Sin embargo, no existe causalidad lineal; la virtualización no es producto de los artefactos. La apertura de un campo problemático y creativo no es inherente a las TICs. EI hipertexto, por tomar un caso, ofrece una fabulosa combinatoria de datos, pero lo virtual sólo emerge cuando el lector, mediante su navegación no-lineal, teje sentidos no predeterminados (Lévy, 1999).

\footnotetext{
${ }^{1}$ Las otras dos dimensiones son llamadas "realización" y "potenciación", pero no hablaremos de ellas aquí por cuanto pertenecen a la realidad considerada como sustancia, no como acontecimiento.
} 
En efecto, la virtualización no surge en cualquier relación, sino específicamente en inter-acciones. En el caso de la incorporación de TICs por parte de los "movimientos sociales", emerge como función del vínculo humano-máquina, y de su intercambio bidireccional de competencias. Otro ejemplo puede aclarar dicha idea:

"Si (...) el desarrollo de un programa informático puramente lógico reemplaza al binomio posible/real, la interacción entre humanos y sistemas informáticos hace lo propio con la dialéctica de lo virtual y lo actual. Previamente, el diseño de un programa, por ejemplo, trata un problema de forma original. Cada equipo de programadores redefine y resuelve de un modo diferente el problema al que se enfrenta. Posteriormente, la actualización del programa a ser utilizado (...), descalifica ciertas competencias y pone en marcha otros motores, activa conflictos, desbloquea situaciones, instaura una nueva dinámica de colaboración, etc. El programa lleva implícita una virtualidad del cambio que el grupo (...) actualiza de manera más o menos imaginativa" (Pág. 19).

Aquí, el software es virtual durante su desarrollo, mientras un colectivo de programadores lo convierte en objeto de su trabajo creativo. Por otro lado, es virtualizante gracias al uso que le da una comunidad específica. El acontecimiento surge cuando los programadores o las comunidades y el software se encuentran y transforman mutuamente; cuando los humanos y los no humanos configuran una red semántica heterogénea, un colectivo que no puede reducirse a las relaciones sociales o a las articulaciones técnicas tomadas independientemente, sino que se define por la mezcla de ambas. Nosotros consideramos que los sistemas y redes de acción se están moviendo en esta dirección: incorporando las TICs se desterritorializan y cuestionan los dualismos local-global, intragrupo-intergrupo, privado-público. Melucci (2001) lo comenta de la siguiente manera:

"Las nuevas tecnologías de la información crean la posibilidad de que la acción se desvincule del espacio y el tiempo, dando así lugar a la presentificación del tiempo y a la virtualización del espacio. Por vez primera en la historia de la humanidad se produce socialmente aquello que sólo el pensamiento mágico había permitido, a saber, la posibilidad de sustraer la acción humana al espacio y el tiempo. El desenclave de la acción social de sus vínculos espacio-temporales y de sus relaciones con el cuerpo implica un salto cualitativo decisivo y acentúa la discontinuidad [respecto a la modernidad] de la que ya he hablado (...) Además las TICs introducen una dimensión de simultaneidad y de globalidad en la experiencia humana que hace perder la jerarquía entre los diversos ámbitos de la acción y hace asimismo, caer las fronteras tradicionales, no sólo las geopolíticas, sino también las perceptivas, las cognitivas y las relacionales" (Pág. 32)

A continuación veamos un caso concreto que ejemplifica estas afirmaciones: se trata del Proveedor de Servicios de Internet para "movimientos sociales" denominado Nodo50. 


\section{Nodo50: Proveedor de Servicios de Internet y Colectivo de Contra-Información}

Nodo50 nació en 1994 como un artefacto articulado al movimiento por la cooperación Norte-Sur. Surgió como una BBS (Bulletin Board System) que daba apoyo telemático al Foro "Las Otras voces del Planeta" celebrado en Madrid, dentro de la campaña "50 años bastan" contra el Fondo Monetario Internacional y el Banco Mundial (Marí Sáez, 2004b; Sadaba \& Roig, 2004). Con posterioridad a dicho evento Nodo50 debía desaparecer, sin embargo, la organización no gubernamental SODePAZ asumió su continuidad y algunos grupos madrileños lo emplearon para conectarse vía e-mail, con colectivos extranjeros.

La mejora de las condiciones técnicas en España y la importancia que cobró Internet en el seno del Movimiento Anti-Globalización (MAG) favoreció el crecimiento y desarrollo de Nodo50. Este proceso incluyó divisiones internas (como la que dio origen a SinDominio) y alianzas con otras organizaciones (como su federación en IPANEX, acrónimo de lepala, Pangea, Altercom, Nodo50, Eusnet, y Xarxaneta); supuso la independencia del mundo de las ONGs y la migración al software al libre, así como momentos de trabajo descentralizado en ausencia de locaciones físicas, etc.

En 1996, Nodo50 dejó de ser una BBS para convertirse en un Proveedor de Servicios de Internet (ISP): un ordenador conectado permanentemente a la Red que ofrece acceso a ciertos servicios; por ejemplo, conectividad, cuentas y listas de correo electrónico, espacio web, gestión y alojamiento de dominios, buscadores, foros, bases de datos y diseño gráfico, entre otros.

La red de relaciones que se tejía en torno a la máquina, también se transformó. Actualmente, Nodo50 es un proyecto autónomo de contrainformación telemática, coordinado por una asamblea independiente. Es una colectividad que reúne a profesionales asalariados y militantes que gestionan el ISP, solucionan problemas, demandas e incidencias de los usuarios. Estos últimos también están integrados en la red. Son miembros de una pléyade de "movimientos sociales" que recurren a Nodo50 como proveedor de servicios, como fuente de contra-información y como espacio de creación. Nodo50 recibe de ellos información, la procesa y la organiza temáticamente, la modera y la distribuye a través de Internet (Sadaba \& Roig, 2004).

En términos de Melucci (1999), podríamos decir que Nodo50 es una "organización de redes". Sin afectar la autonomía de los participantes, ni intentar institucionalizarlos, suministra recursos técnicos y organizacionales para facilitar a los "movimientos sociales" hacerse escuchar en la sociedad; en este caso se trata de colectivos de izquierda y vinculados con el MAG. De esta manera, favorece las condiciones para que los "movimientos sociales" se opongan a la concentración de los medios de comunicación convencionales. En cierto sentido, sólo ofrece soluciones predefinidas: paquetes de servicios por los que se paga una cuota. Las mismas pueden ser consideradas "potencias" en los términos de Lévy (1999), y no virtualizaciones. Pero Nodo50 hace más que eso, ya que propone un modelo alternativo de comunicación que encuentra en Internet su medio paradigmático (Sadaba \& Roig, 2004). Su propuesta es la construcción, gestión y distribución reticular de abundantes cantidades de información que construyen los "movimientos sociales" y que no tiene cabida en los medios dominantes. Dicho modelo genera los siguientes efectos (Sadaba \& Roig, 2004): 1. Visibilización de las agendas de los "movimientos sociales", 2. Creación de nuevas agendas que surgen del encuentro de colectivos diferentes, 3. Coordinación de personas y entidades heterogéneas para mantener en funcionamiento un colectivo o para adelantar iniciativas, 4. Fundación de 
comunidades que comparten un mismo espacio técnico y comunicativo, 5. Formación de identidades globales que surgen de las experiencias compartidas en la red, y, finalmente, 6. Redefinición del territorio de conflicto de los movimientos sociales.

Por esta vía, la experiencia impulsada por Nodo50 con sus organizaciones vinculadas e implementando el uso de las TICs, ha creado nuevas realidades (Sadaba \& Roig, 2004). Particularmente, ha favorecido que el Movimiento Anti-Globalización alcance una cierta autonomía. En términos generales, entendemos al igual que Melucci (1999) que dicho movimiento ocupa una especie de franja intermedia entre las instituciones políticas y la sociedad civil. Como otros "movimientos" contemporáneos, ha alcanzado el estatus de subsistema independiente. Pero, matizamos con Lévy (1999), que dicho acontecimiento emerge por desterritorialización. Las soluciones que ofrece Nodo50 permiten a los "movimientos sociales" desprenderse de la geografía para situarse en un espacio inasignable: el ciberespacio. Allí, la sincronización de los actores reemplaza la unidad de lugar (Lévy, 1999). Ellos ya no se encuentran en un sitio, sino que coinciden en tiempo real. La interconexión, finalmente, sustituye la unidad de tiempo (Lévy, 1999). Diferentes temporalidades y velocidades emergen en virtud del tipo de conexiones que se establecen de los medios empleados y de los actores involucrados.

Lo que queremos dejar claro hasta el momento es que, Nodo50 virtualiza al MAG en la medida en que favorece las interconexiones necesarias para su desterritorialización. Este hecho es posible gracias a relaciones simbióticas entre los "movimientos" y las TICs, así como a relaciones antagónicas con los agentes de la globalización capitalista. El entramado es complejo. Por un lado, las personas se encuentran empleando a las TICs; de este modo, les otorgan sentidos nuevos y las resignifican a través del uso. Pero, a su vez, la tecnología transforma las estructuras organizacionales de los colectivos -inclusive favorece la aparición de comunidades virtuales-, la coordinación trasatlántica de iniciativas y la cohesión misma de grupos locales que se encuentran más frecuentemente on-line que off-line.

Las hibridaciones entre "movimientos" y tecnología no son el único espacio creativo que favorece Nodo50. La oposición a los agentes del capitalismo global también puede considerarse una producción virtual. Sadaba y Roig (2004) lo comentan de la siguiente forma:

"La existencia de interlocutores globales difícilmente identificables en principio, e inalcanzables una vez reconocidos, pero también las dimensiones y la heterogeneidad ideológica del MAG (con una rama propositiva y otra centrada en la protesta), la dificultad para llegar a acuerdos en un programa y, sencillamente, los problemas que supone renegociar todo un orden ideológico, hacen que el conflicto (inherente a cualquier movilización social) tenga que ser replanteado." (Pág. 206).

Para que surja el antagonismo global, el conflicto, es necesario nombrar primero ciertos aspectos de la realidad: determinar un lenguaje, enunciarlo, organizar la realidad de acuerdo a un código, negociar constantemente los recursos, las finalidades y las limitaciones del sistema de acción, es decir, construir una identidad colectiva (Melucci, 1999). Esto precisamente es lo que favorece Nodo50. En su portal (www.nodo50.org) es posible encontrar referencias a dicha identidad. Citamos a continuación un par de ejemplos:

"La lucha contra los efectos sociales de la mundialización económica hace ya tiempo que se convirtió en acción colectiva globalizada. Sólo la actuación dentro de una red mundial hace posible que los movimientos sociales actúen como oposición 
política con capacidad de intervención real frente a las estructuras de poder económico y a los discursos hegemónicos. Hoy en día gran parte de la legitimidad de los movimientos sociales se basa en su credibilidad como productores de informaciones fiable. De ahí que aceptemos jugar un juego cuya lógica denunciamos: resistir a las estructuras del discurso único hablando en lugar de ser hablados, generando nuevos discursos y con ellos nuevas realidades, ampliando las luchas reales desde lo virtual. (...) Nuestro objetivo es crear un polo de referencia político en la red que se desmarque del ruido, el exceso y la saturación que imperan, que promueva usos sociales y no comerciales de las nuevas tecnologías. Buscamos la visibilidad de la izquierda y la acción colectiva frente al consumo, la intoxicación ideológica y la caza de brujas en Internet. Contrainformamos con la idea de resistir a la apisonadora (sic) ideológica que ha puesto en marcha el capitalismo postmoderno, con la ilusión puesta en demostrar que aún estamos, resistimos y, como no, nos preparamos para la victoria."

Nodo50 está preocupada por conformar una identidad global con la que el MAG pueda enfrentarse a antagonistas también planetarios. Tal enfrentamiento, por otro lado, no se presenta como una confrontación "real", sino que pasa a un plano simbólico: a la creación de una controversia pública y la defensa de su relevancia (Sadaba \& Roig, 2004). Lo que llevaría a cuestionarnos ¿cómo se expresará la violencia implícita en todo conflicto social si el conflicto pasa al plano de lo simbólico, cuando es precisamente este desplazamiento al nivel simbólico una nueva característica de muchos movimientos? Estamos hablando aquí de la visibilización de un conflicto empleando Internet, pero adicionalmente, de su configuración mediante la construcción de identidades. Aludimos, finalmente, a un movimiento de carácter simbólico: la estructuración conflictiva de un mensaje, su distribución social, sus efectos aglutinantes y antagónicos, su capacidad para producir realidades, y generar tensiones. Si pensamos en la forma como este mensaje es tejido encontraremos la respuesta a nuestra inquietud ¿qué se mueve en los "movimientos sociales"?

\section{¿Qué se mueve en los "movimientos sociales"?}

En esta comunicación hemos pasado de la idea de "movimiento social" como dato, al concepto de "sistema de acción" como logro. Este logro, por otro lado, no es un suceso. Los estudios basados en sucesos, nos dice Melucci (1999), "están relacionados con el efecto final de la acción no con la forma en que la acción se produce" (Pág. 61). Nosotros proponemos ver la acción social, entonces, como "acontecimiento".

Los acontecimientos no son productos, ni son episodios meramente públicos. Son procesos dinámicos que devienen entre un polo latente de invención de problemas (la virtualización) y un polo manifiesto de resolución innovadora (la actualización). Dichas dimensiones creativas de la realidad favorecen desconexiones del aquí y el ahora (desterritorializan) y hacen frágiles las fronteras entre lo global y lo local, lo público y lo privado, lo interior y lo exterior.

A través de un ejemplo, hemos visto algunas formas como el acontecimiento emerge en la simbiosis entre "movimientos sociales" y TICs. En el segmento anterior, apreciamos que Nodo50, el MAG, los agentes de la globalización capitalista, y las redes telemáticas, se encuentran anudados en un inmenso colectivo donde emerge la virtualización en al menos, tres formas: 1. Como 
desterritorialización del Movimiento-Antiglobalización; 2. Como una identidad colectiva negociada y negociable, y 3. Como creación de un campo conflictivo novedoso cuyas dimensiones son planetarias. En cierto sentido, estas tres virtualizaciones están secuencialmente encadenadas. Son progresivamente dependientes la una de la otra. Pero existe un elemento que las atraviesa a todas. Un objeto que, mientras circula socialmente, dibuja los campos de atracción o de antagonismo de este sistema interactivo. Nos referimos a la información, especialmente, a la que hoy en día irrumpe en forma digital.

Al principio de esta comunicación definimos la "información" como el contenido y los procesos de conocimiento y de acción social que surgen en el contexto de las interacciones entre personas y grupos. Una vez hecho el recorrido por los aportes de Melucci y de Lévy, así como el examen de Nodo50 como caso, podemos afirmar que las redes de solidaridad e interacción son información.

La acción de "informar", no ocurre sólo "en el contexto de las interacciones". La información es texto, pero es también tejido: objeto y vínculo. Desde Lévy, la idea de los "sistemas de acción" tiene pleno sentido, ya que podemos entenderlos a la vez como estructuras sociales, y como mensajes. Objeto y enlace se constituyen mutuamente, y surgen simultáneamente: la información tiene sentido en un contexto de producción colectiva, y dicho contexto encuentra en la información su sentido. "Informar" es, entonces, ese proceso virtualizante que da origen a los colectivos-mensaje, a los "colectivos inteligentes", donde estructura y sentido son inseparables.

La Sociedad de la Información, finalmente, es el resultado de la desterritorialización de la acción informativa de dichos colectivos, de su trabajo por superar la diferencia local-global, dentro-fuera, privado-público. El poder en esta forma de sociedad, es la capacidad de dar forma a la realidad organizando el flujo de información. Lo cual significa para Lévy (1999), poner obstáculos a la potencia. Es decir, moldear los procesos de virtualización, condicionarlos, limitarlos. Las TICs pueden operar de esta manera, pero pueden ser empleadas también en sentido opuesto: para abrir márgenes de libertad, nuevos horizontes. El trabajo de los "movimientos sociales" en este sentido es paradigmático. De esta manera, a lo que nos enfrentamos cuando hablamos de Sociedad de la Información es a un campo problemático. En él, la información ha tejido unas ciertas redes de solidaridad que se encuentran en el escenario global: relaciones sociales en general, sistemas políticos y sistemas de acción en particular, colectivos inteligentes que tratan de circular como mensajes. Como ya hemos afirmado, no todos tienen las mismas condiciones para hacerlo, pero la naturaleza del nuevo objeto (la información), cuyo flujo no puede ser fácilmente controlado, está abriendo la posibilidad para cambiar este estado de cosas, está virtualizando los "movimientos sociales", los está haciendo "glocales" y desterritorializados.

Es el momento entonces de comentar qué ha ocurrido hasta aquí con la noción de movimiento. Pues que hemos pasado de emplearla como una palabra que se refiere a un actor, para convertirse en una especie de actor-red, esto es, un proceso de establecimiento y negociación constante de relaciones, en virtud de la circulación de un objeto. Hay algo que, efectivamente, se mueve en los "movimientos sociales". Pero no son personas con pancartas, o multitudes enloquecidas por el pánico, tampoco son grupos organizados para la acción antagónica. Lo que se mueve son colectivos-mensaje. Lo que circula es la información. 


\section{Referencias}

CONTRERAS, Pau (2003). Me llamo Kohfam. Identidad hacker: una aproximación antropológica. Barcelona (España): Editorial Gedisa, 2003.

GERGEN, Kenneth (1991). El yo saturado. Dilemas de identidad en el mundo contemporáneo. Traducción de Leandro Wolfson. $3^{\mathrm{a}}$ reimpresión. Barcelona (España): Ediciones Paidós Ibérica, 1997.

HIMANEN, Pekka (2001). La ética hacker el espíritu de la era de la información. Traducción de Ferran Meler Ortí. Barcelona: Editorial Destino, 2002.

LEVY, Pierre (1995). ¿Qué es lo virtual? Traducción de Diego Levis. Barcelona: Ediciones Paidós, 1999.

MARÍ SÁEZ, Víctor Manuel (2004a). Cuando los movimientos sociales se apropian de la red. En MARÍ SÁEZ, Víctor (coord.) (2004). La red es de todos. Cuando los movimientos sociales se apropian de la red. Madrid: Editorial Popular, 2004.

MARÍ SÁEZ, Víctor Manuel (2004b). Comunicación, redes y cambio social. En MARÍ SÁEZ, Víctor (coord.) (2004). La red es de todos. Cuando los movimientos sociales se apropian de la red. Madrid: Editorial Popular, 2004.

MELUCCI, Alberto (1996). Challenging Codes. Collective action in the information age, Cambridge, Cambridge University Press.

MELUCCI, Alberto (1999). Acción Colectiva, Vida Cotidiana y Democracia. El Colegio de México, Centro de Estudios Sociológicos 1999.

MELUCCI, Alberto (2001). Vivencia y convivencia. Teoría social para una era de la información. Madrid: Editorial Trotta, 2001.

SADABA, Igor \& ROIG, Gustavo (2004). Nodo50. Territorio virtual para los movimientos sociales y la acción política. En MARÍ SÁEZ, Víctor (coord.) (2004). La red es de todos. Cuando los movimientos sociales se apropian de la red. Madrid: Editorial Popular, 2004.

ZUBERO, Imanol (2004). Conocer para hacer: la tarea cultural de los movimientos sociales. En MARÍ SÁEZ, Víctor (coord.) (2004). La red es de todos. Cuando los movimientos sociales se apropian de la red. Madrid: Editorial Popular, 2004 